\title{
Potential anticancer effect of aspirin and 2'-hydroxy-2,3,5'-trimethoxychalcone-linked polymeric micelles against cervical cancer through apoptosis
}

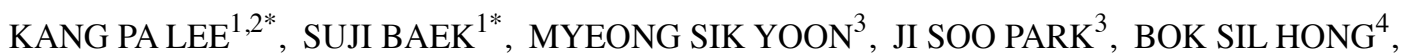 \\ SANG JU LEE ${ }^{5}$, SEUNG JUN OH ${ }^{5}$, SEUNG HAE KWON ${ }^{6}$, RUDA LEE ${ }^{7}$, \\ DAE HO LEE ${ }^{8}$, KANG-SEO PARK ${ }^{8}$ and BYUNG SEOK MOON ${ }^{2}$
}

\begin{abstract}
${ }^{1}$ Research and Development Center, UMUST R\&D Corporation, Seoul 01411; ${ }^{2}$ Department of Nuclear Medicine, Ewha Womans University College of Medicine, Seoul 07804; ${ }^{3}$ Department of Pharmaceutical Engineering, Hoseo University, Cheonan, Chungnam 31499; ${ }^{4}$ Department of Nursing, Cheju Halla University, Jeju 63092;

${ }^{5}$ Department of Nuclear Medicine, Asan Medical Center, University of Ulsan College of Medicine, Seoul 05505; ${ }^{6}$ Seoul Center, Korean Basic Science Institute, Seoul 02841, Republic of Korea; ${ }^{7}$ International Research Organization for Advanced Science and Technology, Kumamoto University, Kumamoto, Japan; ${ }^{8}$ Department of Oncology, Asan Medical Center, University of Ulsan College of Medicine, Seoul 05505, Republic of Korea
\end{abstract}

Received July 5, 2021; Accepted November 5, 2021

DOI: $10.3892 / 01.2021 .13149$

\begin{abstract}
Although early diagnosis and treatment of cancers in women are achievable through continuous diagnostic tests, cervical cancer (CVC) still has a high mortality rate. In the present study, we investigated whether certain nanoparticles (NPs), comprising aspirin conjugated 2'-hydroxy-2,3,5'-trimethoxychalcone chemicals, could induce the apoptosis of cancer cells. HeLa cells were treated with NPs and the cell viability was evaluated using WST-1 assay. Protein expression of Ki-67 was measured using immunocytochemistry. In addition, the apoptotic effect of NPs was determined using TUNEL assay. To investigate the apoptosis signaling pathways, reverse transcription quantitative PCR was performed and lipid accumulation was observed via holotomographic microscopy. The $\mathrm{IC}_{50}$ value of the NPs was $4.172 \mu \mathrm{M}$ in HeLa cells. Furthermore, $10 \mu \mathrm{M}$ NPs significantly inhibited the cell proliferation and stimulated the apoptosis of HeLa cells.
\end{abstract}

Correspondence to: Professor Kang-Seo Park, Department of Oncology, Asan Medical Center, University of Ulsan College of Medicine, 88 Olympic-ro 43-Gil, Songpa-gu, Seoul 05505, Republic of Korea

E-mail: 77kangseo@gmail.com

Professor Byung Seok Moon, Department of Nuclear Medicine, Ewha Womans University College of Medicine, 260 Gonghang-daero, Gangseo-gu, Seoul 07804, Republic of Korea

E-mail: bsmoon@ewha.ac.kr

*Contributed equally

Key words: anticancer, micelle, apoptosis, mitochondria, lipid
In addition, apoptosis and mitochondrial dysfunction were induced by the NPs through lipid accumulation in HeLa cells, leading to apoptotic signaling cascades. Taken together, the results from the present study demonstrated that the NPs developed promoted apoptosis though efficient lipid accumulation in HeLa cells, suggesting that they may provide a novel way to improve the efficacy of CVC anticancer treatment.

\section{Introduction}

Cervical cancer (CVC) is currently the second leading cause of mortality in women worldwide, and according to the in 2018 World Health Report of the World Health Organization, the average age-adjusted incidence of CVC cancer was 13.1 per 100,000 (1). Morbidity and mortality rates are higher in developing countries compared with developed countries (2). Vaccination against the human papillomavirus (HPV) can reduce the incidence of CVC, although CVC cannot be completely prevented (3). Further research is therefore needed for the development of CVC treatment strategies beyond the methods used for CVC diagnosis such as smear cytology and colposcopy.

CVC is highly associated with oncogenic mutations caused by HPV infection, which are responsible for host cell proliferation (4). Furthermore, most HPV types, including HPV16, HPV18 and HPV84, encode oncoproteins such as E6 and E7 that overcome host tumor-suppressor and cell-cycle checkpoint proteins like p53, cyclin E, p21, and p27 (5-8). Current treatments for CVC include surgery, radiation therapy and chemotherapy $(9,10)$. In the case of chemotherapy, certain drugs such as cisplatin and doxorubicin are preferred for reducing the preoperative tumor size or preventing postoperative recurrence, as well as for treating metastases (11). However, some patients have reported some adverse side effects or presented with resistance to drug therapy $(12,13)$. 
Apoptosis is one of the main mechanisms of action of chemotherapeutic cancer drugs and is a type of mitochondria-mediated cell death (14). Apoptotic stress activates pro-apoptotic agents such as BAX, which are regulated by the activated Bcl-2 molecule. Activated BAX stimulates the release of cytochrome $c$ from the mitochondria. In addition, the released cytochrome $c$ induces apoptosis by proteolysis, membrane damage and DNA cleavage through activation of Apaf-1, caspase-9 and caspase-3 (15). In particular, oxidative stress-induced cell-death pathways promote mitochondrial fission, which is associated with lipid accumulation $(16,17)$. In addition, doxorubicin can induce lipid droplet accumulation in the cytoplasm in various types of cancer, including colorectal cancer, lymphoma and breast cancer, which participate in apoptosis (18). One important strategies for treating CVC may therefore involve the induction of apoptosis by regulating mitochondrial function.

Recently, aspirin has been used for its anticancer effects in various types of tumor, including gastric, esophageal and colon cancers (18). For example, in gastric cancer cell line SGC7901, the survival of cells treated with 3.0 and $10.0 \mathrm{mmol} / 1$ aspirin for $24 \mathrm{~h}$ was decreased by 44.6 and $88.5 \%$, respectively (19). Data from a clinical report indicated that aspirin may help inhibiting breast cancer metastasis $(20,21)$. Subsequently, in a previous study, we designed an aspirin conjugated chalcone derivative that had anti-inflammation and anti-tumor properties and prepared nanoparticle (NP) formulations, since some anticancer drugs, including the chemical used in the present study, have non-polar properties that make them difficult to be used in an injectable form (22). This revealed that aspirin and 2'-hydroxy-2,3,5'-trimethoxychalcone linked polymeric nanoparticles (AS-DK143-NPs) selectively targeted cancer cells and significantly reduce tumor sizes in a breast cancer xenograft model with low toxicity to healthy tissues. In the present study extended our previous observation by performing additional experiments to provide a comprehensive understanding of the role of AS-DK143-NPs against CVC in several representative solid cancer cell lines such as HepG2, BXPC-3, AGS and HeLa.

\section{Materials and methods}

Reagents. Dulbecco's modified Eagle's medium (DMEM), Roswell Park Memorial Institute (RPMI) medium, fetal bovine serum (FBS) and penicillin/streptomycin (P/S) were purchased from Welgene Inc. WST-1 and bovine serum albumin (BSA) were purchased from Sigma-Aldrich; Merck KGaA. A TUNEL assay kit was obtained from Promega Corporation. Hoechst dye, Mito-tracker ${ }^{\mathrm{TM}}$ Red CMXRos and the Alexa-Fluor 488-conjugated donkey anti-Rabbit antibody were purchased from Thermo Fisher Scientific, Inc. The anti-Ki-67 antibody was purchased from Abcam (cat. no. ab15580). The aspirin conjugated DK143 [(E)-2-(4-(2,3-dimethoxyphenyl) acryloyl)-4-methoxyphenyl-2-acetoxybenzoate, AS-DK143] and AS-DK143-loaded methoxy poly(ethylene glycol)-poly(L-lactide) NPs (AS-DK143-NPs) were prepared and characterized according to our previous report (22). HeLa cell line, HepG2, which is a hepatoblastoma cell line, and AGS cell line were purchased from the Korean Cell Line Bank; Korean Cell Line Research Foundation. BXPC-3 cells were obtained from the Japanese Collection of Research Bioresources Cell Bank.

Cell line authentication. Cell lines were authenticated using short tandem repeat (STR) analysis as described in 2012 in the ANSI standard (ASN-0002) by the American Type Culture Collection Standards Development Organization and by Reid et al (23) The match criterion is based on an algorithm that compares the number of shared alleles between the reference and sample profile.

Preparation of (E)-2-(4-(2,3-dimethoxyphenyl) acryloyl)-4-methoxyphenyl-2 acetoxybenzo ate (AS-DK143)-loaded polymeric micelles. (E)-3-(2,3-dimethoxyphenyl)-1-(2-hydroxy-5-methoxyphenyl) prop-2-en-1-one (DK143), one of the chalcone derivatives, was used to synthesize AS-DK143 using the following procedures. 1-(3-Dimethylaminopropyl)-3-ethylcarbodimide hydrochloride (EDC $\cdot \mathrm{HCl}, 0.641 \mathrm{~g}, 3.3 \mathrm{mmol}$ ) and 4-dimethylaminopyridine (DMAP, $0.816 \mathrm{~g}, 3.3 \mathrm{mmol}$ ) were added to chloroform $(50 \mathrm{ml})$ containing aspirin $(0.574 \mathrm{~g}, 3 \mathrm{mmol})$ and DK143 (1.0 g, $3 \mathrm{mmol})$. The solution was stirred at room temperature for $24 \mathrm{~h}$. At the end of the reaction, ice water was added to the mixture solution and acidified with $6 \mathrm{~N} \mathrm{HCl}$. After extraction with ethyl acetate $(100 \mathrm{ml}, \mathrm{x} 3)$, the organic layer was purified using silica gel flash column chromatography to yield $33.3 \%(0.48 \mathrm{~g})$ as a pale-yellow solid.

Preparation of AS-DK143-loaded mPEG-PLA nanoparticles (NPs). AS-DK143 polymeric micelles (AS-DK143-NPs) were prepared using the thin-film hydration method. Briefly, methoxy poly(ethylene glycol)-b-poly(D,L-lactide) (mPEG-PLA, $60 \mathrm{mg}$ ) and AS-DK143 (10 mg) were dissolved in dichloromethane $(50 \mathrm{ml})$ and then removed by rotary evaporator under reduced pressure at $40^{\circ} \mathrm{C}$. The film obtained from this process contained AS-DK143 distributed in an amorphous form. The film was continuously hydrated in distilled water $(50 \mathrm{ml})$ at room temperature. The solution was filtered through a syringe filter $(0.2 \mu \mathrm{m})$ and lyophilized for $48 \mathrm{~h}$.

Cell viability and cell morphology. HeLa (human cervical cancer cell line) and HepG2 cells (human liver cancer cell line) were cultured in DMEM supplemented with $10 \%$ FBS and $1 \% \mathrm{P} / \mathrm{S}$ and placed at $37^{\circ} \mathrm{C}$ in a humidified incubator containing $5 \% \mathrm{CO}_{2}$. AGS (human gastric adenocarcinoma hyperdiploid cell line) and BXPC-3 cells (human pancreatic cancer cell line) were cultured in RPMI supplemented with $10 \% \mathrm{FBS}$ and $1 \% \mathrm{P} / \mathrm{S}$ and placed at $37^{\circ} \mathrm{C}$ in a humidified incubator containing $5 \% \mathrm{CO}_{2}$. Cells were seeded at the density of $1 \times 10^{4}$ cells/well in 96-well plates, cultured for $24 \mathrm{~h}$ and treated with different concentration of AS-DK143-NPs $(1,3,10,30,100$ or $300 \mu \mathrm{M})$ for $24 \mathrm{~h}$. To determine the cell viability, cells were incubated with $10 \mu \mathrm{l}$ of WST-1 for $2 \mathrm{~h}$ at $37^{\circ} \mathrm{C}$. Absorbance was read at $450 \mathrm{~nm}$ using an iMARK spectrophotometer (Bio-Rad Laboratories, Inc.). Cell-morphology images were captured using an inverted microscope (magnification, x200; Nikon Corporation).

TUNEL assay. HeLa cells were plated in 8-chamber slides at a density of $0.5 \times 10^{4}$ cells/well, and the cells were treated with 


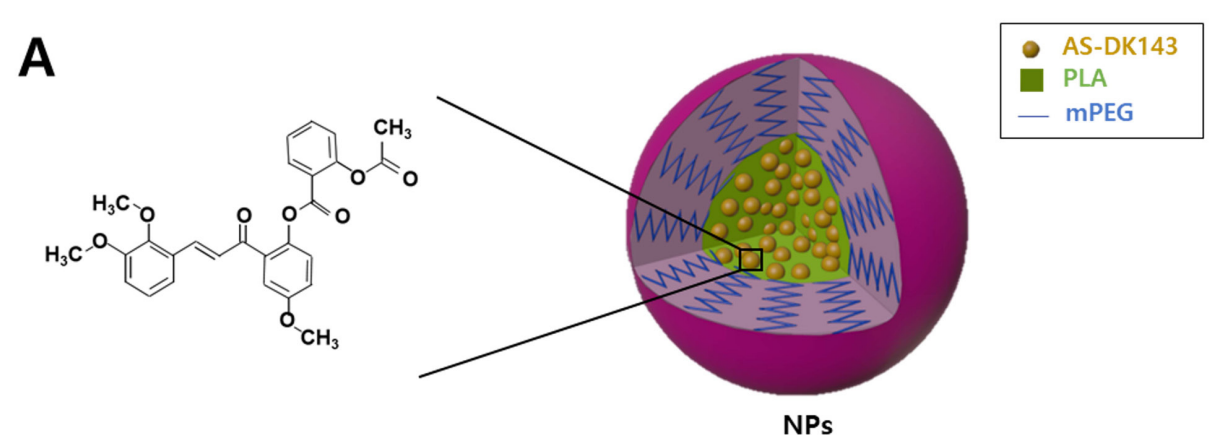

B

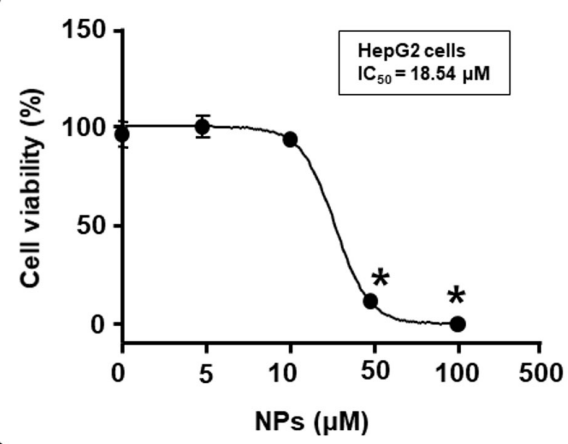

D

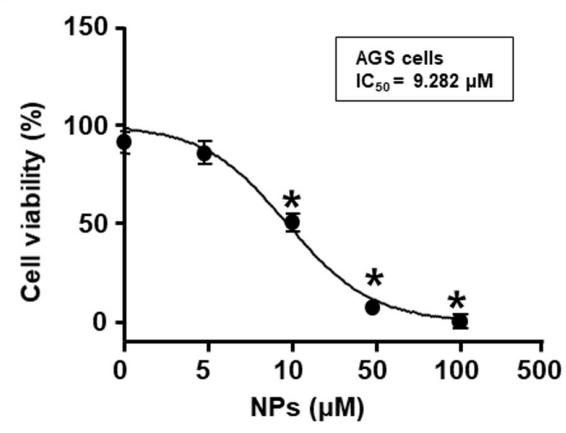

C

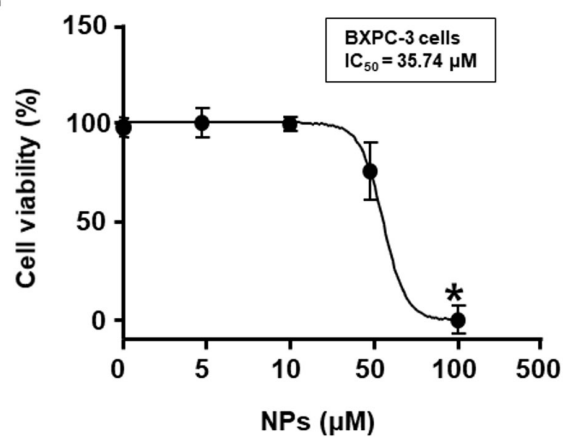

E

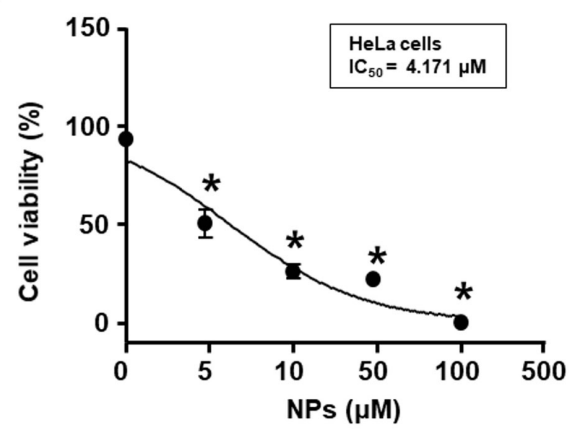

Figure 1. Anticancer effects of AS-DK143 in various types of cancer cell. (A) Schematic representation of AS-DK143-NPs. (B) HepG2, (C) Bxpc-3, (D) AGS and (E) HeLa cells were treated with various concentrations $(1,3,10,30,100$ or $300 \mu \mathrm{M})$ of NPs for $24 \mathrm{~h}$. Cell viability was evaluated using WST-1 assay. ${ }^{*} \mathrm{P}<0.05$ vs. untreated group. AS, aspirin; NPs, nanoparticles; mPEG-PLA, methoxy poly(ethylene glycol)-b-poly(D,L-lactide).

AS-DK143-NPs for $24 \mathrm{~h}$. Cells were fixed in $4 \%$ paraformaldehyde (PFA) and permeated in $0.1 \%$ TritonX-100 solution for $5 \mathrm{~min}$ at room temperature. Cells were incubated with equilibration buffer for $5 \mathrm{~min}$ and treated with recombinant terminal deoxynucleotidyl transferase (rTdT) reaction solution (consisting of equilibration buffer, a mixture of biotinylated nucleotides, and rTdT enzyme) for $1 \mathrm{~h}$ at $37^{\circ} \mathrm{C}$. The cells were immersed in $2 \mathrm{X}$ SSC buffer $(\mathrm{NaCl}$ and sodium citrate) and washed with phosphate-buffered saline. The cells were incubated with streptavidin-conjugated horseradish peroxidase solution for $30 \mathrm{~min}$. The cells were reacted with 3,3'-diaminobenzidine (DAB) solution (DAB chromogen and DAB substrate). Subsequently, the cells were mounted and observed under a light microscope.

Immunocytochemistry. HeLa cells $\left(1 \times 10^{5}\right.$ cells/well) were seeded in 30-mm dishes and treated with AS-DK143-NPs for $24 \mathrm{~h}$. Cells were then fixed with $4 \%$ PFA for $10 \mathrm{~min}$ at room temperature (RT) and permeabilized using 0.1\% Triton-X 100 for $15 \mathrm{~min}$ at RT. The cells were blocked for $1 \mathrm{~h}$ in $5 \%$ BSA at room temperature and incubated with a primary antibody against Ki-67 $(1: 1,000)$ for $24 \mathrm{~h}$ at $4^{\circ} \mathrm{C}$. Subsequently, cells were incubated for $1 \mathrm{~h}$ at RT with a fluorophore-conjugated secondary antibody $(1: 1,000)$. Cells were further incubated with Hoechst solution for $20 \mathrm{~min}$ at room temperature. Fluorescence was observed using a K1-fluo microscope (magnification, x200; Nanoscope Systems, Inc.).

Mitochondrial labeling. HeLa cells were seeded in 30-mm confocal dishes at the density of $1 \times 10^{5}$ cells/well and were treated with 10 and $30 \mu \mathrm{M}$ of AS-DK143-NPs for $24 \mathrm{~h}$. Cells were loaded with $500 \mathrm{nM}$ Mito-tracker ${ }^{\mathrm{TM}}$ Red CMXRos (excitation: $579 \mathrm{~nm}$, emission: $599 \mathrm{~nm}$ ) for $30 \mathrm{~min}$ at $37^{\circ} \mathrm{C}$. The medium was replaced and mitochondrial images were obtained using a Zeiss KSM 780 microscope (magnification, 400; Ziess GmbH).

Reverse transcription quantitative $(R T-q) P C R$. Total RNA was isolated from HeLa cells using TRIzol reagent (Thermo Fisher Scientific, Inc.) and mixed with chloroform. The resulting supernatant was incubated with isopropanol for $20 \mathrm{~h}$ at $4^{\circ} \mathrm{C}$ and then centrifuged at $8,000 \mathrm{x} \mathrm{g}$ for $15 \mathrm{~min}$ at $4^{\circ} \mathrm{C}$. The total RNA was quantified using a NanoDrop device (NanoDrop $^{\text {TM }}$ 2000/2000c spectrophotometer; Thermo Fisher Scientific Inc.). cDNA was synthesized using $1 \mu \mathrm{g}$ RNA and 
A
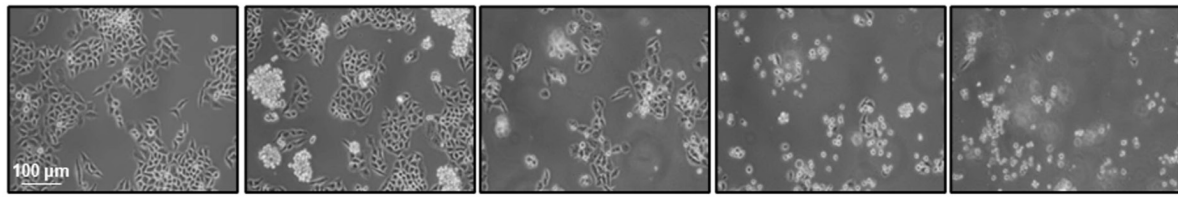

Veh 10 100 300

NPs $(\mu \mathrm{M})$
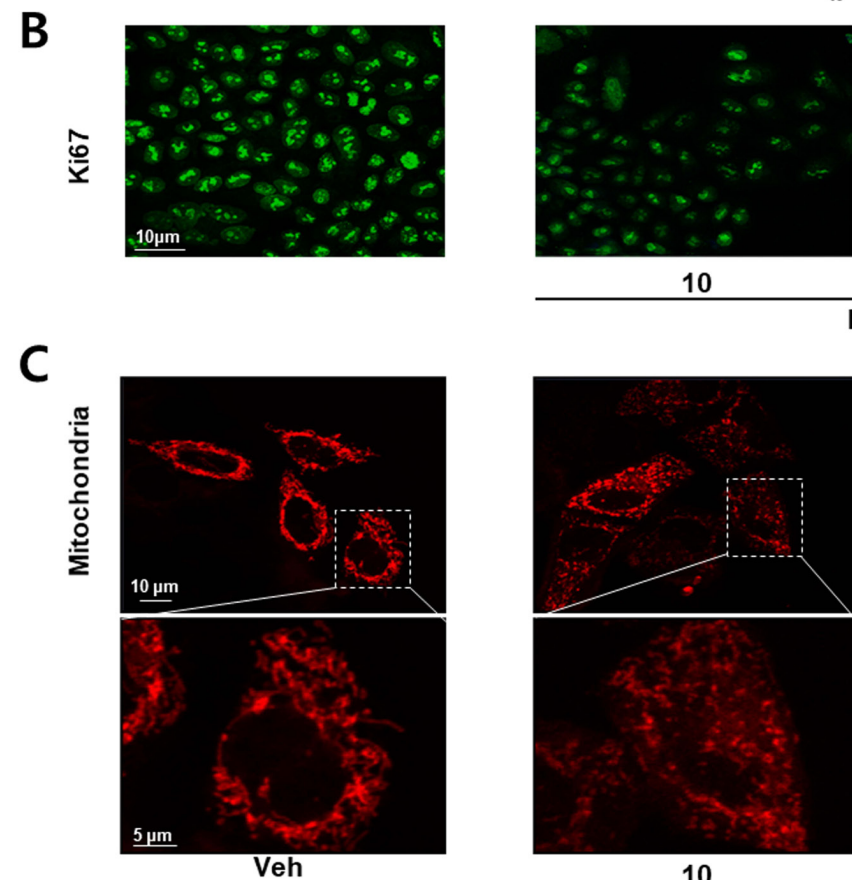

10

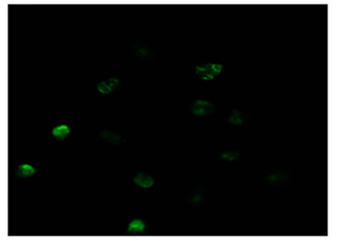

30

NPs $(\mu \mathrm{M})$

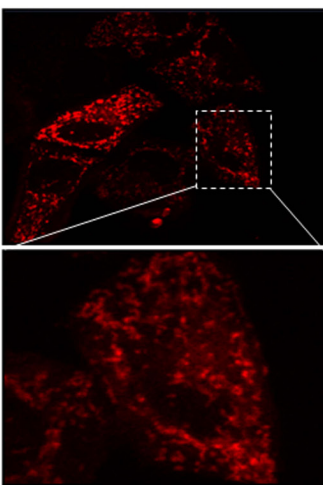

10
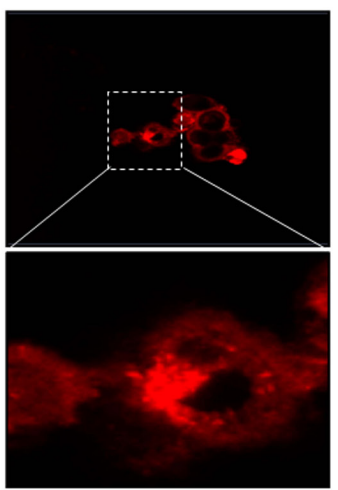

30

NPs $(\mu \mathrm{M})$

Figure 2. AS-DK143-NPs induces HeLa cell damage. Cells were treated with 10, 30, 100 and $300 \mu \mathrm{M}$ of AS-DK143-NPs for 24 h. (A) Cell morphology was observed using light microscopy. Scale bar=100 $\mu \mathrm{M}$. (B) Ki-67 expression was detected by fluorescence microscopy. Scale bar=10 $\mu \mathrm{M}$. (C) Mitochondria were stained with Mito-trackerTM Red CMXRos. Scale bars=5 and $10 \mu \mathrm{M}$. Veh, vehicle; NPs, nanoparticles; AS, aspirin.

the Superscript III First Strand cDNA Synthesis Kit. qPCR was performed on an Exicyler ${ }^{\mathrm{TM}} 96$ instrument (Bioneer Corporation) using SYBR master mix (Bioneer Corporation). The following PCR thermocycling conditions were used: Initial denaturation at $95^{\circ} \mathrm{C}$ for $10 \mathrm{~min}, 40$ cycles of denaturation for $10 \mathrm{sec}$ at $95^{\circ} \mathrm{C}$ and annealing at $60^{\circ} \mathrm{C}$ for $30 \mathrm{sec}$, followed by a final extension step for $30 \mathrm{sec}$ at $72^{\circ} \mathrm{C}$. The sequences of the primers were as follows: CD36 forward, 5'-TGGAACAGAGGCTGACAACTT-3', reverse, 5'-TTGATT TTGATAGATATGGGATGC-3'; BAX forward,5'-GCGTCCA CCAAGAAGCTGAG-3', reverse, 5'-ACCACCCTGGTCTTG GATCC-3'; Bcl2 forward, 5'-TGTGGCCTTCTTTGAG TTCG-3', reverse, 5'-TCACTTGTGGCCCAGATAGG-3'; p53 forward, 5'-TGTGGAGTATTTGGATGACA-3', reverse, 5'-GAACATGAGTTTTTTATGGC-3'; and $\beta$-actin forward, 5'-GTGATGGTGGGCATGGGTC-3' and reverse, 5'-ACGGC CAGAGGCGTACAGGG-3'. The relative expression levels were normalized to endogenous control $\beta$-actin and were expressed as $2^{-\Delta \Delta \mathrm{Cq}}(24)$.

3-dimension (3D) holotomography. To detect the effects of AS-DK143-NPs on live cells, HeLa cells $\left(1 \times 10^{5}\right.$ cells/well $)$ were seeded in $60-\mathrm{mm}$ Tomodishes and treated with $30 \mu \mathrm{M}$ AS-DK143-NPs at $36^{\circ} \mathrm{C}$ for 30, 60 and $120 \mathrm{~min}$.
3D holotomography images of live cells were obtained using an HT-1 microscope (magnification, x600; Tomocube, Inc.). The cell structures were scanned using a digital micromirror device and reconstructed using a 3D refractive index tomogram. The masses of the cytoplasm and lipid droplets were analyzed based on densities of $0.135 \mathrm{~g} / \mathrm{ml}$ and $0.178 \mathrm{~g} / \mathrm{ml}$, respectively.

Statistical analysis. Data are expressed as the means \pm standard deviation of at least three independent experiments. Statistical analyses were performed using GraphPad Prism software version 4.0 (GraphPad Software, Inc.). Data were compared using Student's t-test and one-way ANOVA followed by with Tukey's post hoc test. $\mathrm{P}<0.05$ was considered to indicate a statistically significant difference.

\section{Results}

Effect of AS-DK143 on different human cancer cell lines. Fig. 1 shows the chemical structure of AS-DK143 and the formed NPs that were used as an efficient drug delivery system. Cell viability assay was performed to evaluate the anti-tumor effects of AS-DK143-NPs on the four cell lines HepG2, BXPC-3, AGS and HeLa. Cells were treated with 
A
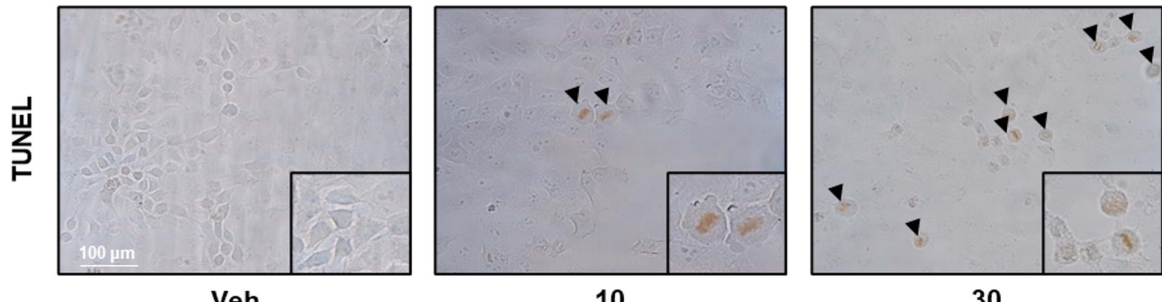

10

NPs $(\mu \mathrm{M})$

B
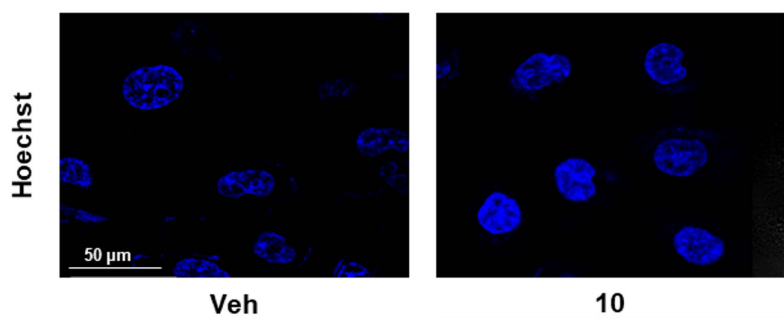

10

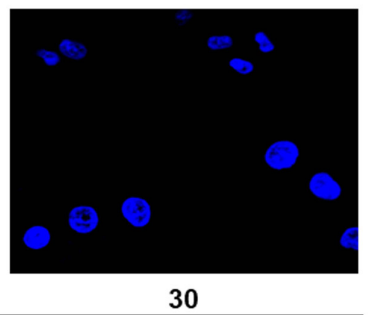

NPs $(\mu M)$

C
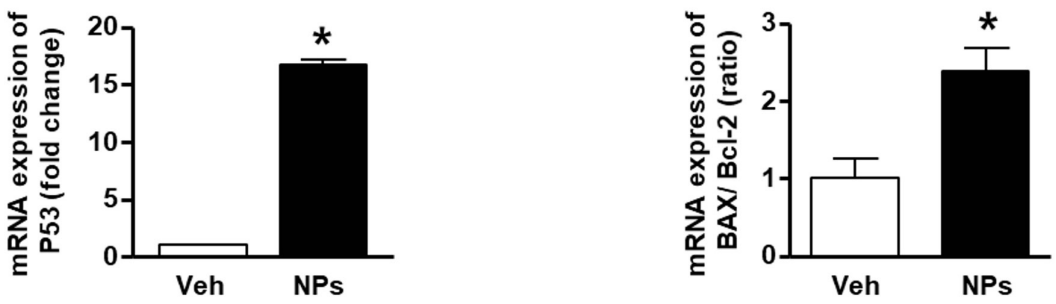

Figure 3. AS-DK143-NPs induces apoptosis in HeLa cells. Cells were treated with or without AS-DK143-NPs (10 and $30 \mu$ M) for 24 h. (A) Apoptotic cells were detected by TUNEL assay. Block arrows indicate the apoptotic cells. Scale bar $=100 \mu \mathrm{M}$. (B) Nuclei were stained with Hoechst 33342 dye. Representative images were captured using confocal microscopy. Scale bar=50 $\mu \mathrm{M}$. (C) mRNA-expression levels of p53, BAX and Bcl-2 evaluated by reverse transcription quantitative PCR. "P<0.05 vs. untreated group. Veh, vehicle; NPs, nanoparticles; AS, aspirin.

increasing concentration of AS-DK143 for $24 \mathrm{~h}$. The inhibitory rate was determined using WST-1 assay. As presented in Fig. 2, the $\mathrm{IC}_{50}$ values of AS-DK143-NPs were 18.54, $35.74,9.28$ and $4.17 \mu \mathrm{M}$ in HepG2, BXPC-3, AGS and HeLa cells, respectively. The AS-DK143-NPs revealed a reducing effect on cell viability in HeLa cells compared with other cell lines.

Anticancer effects of AS-DK143-NPs on HeLa cells. To determine the anticancer effects of AS-DK143-NPs, we performed cell morphology and immunocytochemistry assays. As presented in Fig. 2A, cells were treated with different concentrations of AS-DK143-NPs (10, 30, 100 and $300 \mu \mathrm{M}$ ) for $24 \mathrm{~h}$. AS-DK143-NPs treatment induced changes in cell morphology and viability. Subsequently, the anticancer effects of AS-DK143-NPs were confirmed by studying cell proliferation arrest using Ki-67 antibody for immunocytochemical staining. As presented in Fig. 2B, AS-DK143-NPs treatment decreased Ki-67 expression in HeLa cells. In addition, AS-DK143-NPs induced mitochondrial fission (Fig. 2C).

AS-DK143-NPs induces apoptosis in HeLa cells. To determine whether AS-DK143-NPs could induce apoptosis in HeLa cells, TUNEL assays, Hoechst staining and RT-qPCR were performed. HeLa cells were grown in the absence or presence of AS-DK143-NPs (10 and $30 \mu \mathrm{M})$ for $24 \mathrm{~h}$. The results demonstrated that AS-DK143-NPs increased TUNEL-positive staining in a concentration dependent manner, whereas the vehicle control-treated cells were not stained with the TUNEL solution (Fig. 3A). In addition, AS-DK143-NPs induced nucleolus aggregation in HeLa cells (Fig. 3B). Furthermore, we confirmed that AS-DK143-NPs induced HeLa apoptosis by evaluating the mRNA expression levels of certain apoptosis-related genes. As presented in Fig. 3C, AS-DK143-NPs significantly increased the expression level of p53 and the BAX/Bcl-2 ratio.

AS-DK143-NPs induces lipid accumulation in HeLa cells. To determine whether AS-DK143-NPs could induce lipid accumulation, we performed live cell imaging, 3-D holographic imaging using a holotomographic microscope and determined CD36 mRNA expression by RT-qPCR. Hela cells were treated with AS-DK143-NPs $(10 \mu \mathrm{M})$ for $2 \mathrm{~h}$ and 2D images representing their cell morphology were taken (Fig. 4A). We observed the formation of apoptotic bodies over time. The $3 \mathrm{D}$ images revealed lipid droplet formation (red color) and lipid accumulation occurred in a time-dependent manner. The 3D holotomographic-imaging assay was performed to evaluate lipid masses in live cells. The results demonstrated that lipid accumulation was not altered in vehicle treated cells but was significantly increased ( 6 -fold) in HeLa cells treated 

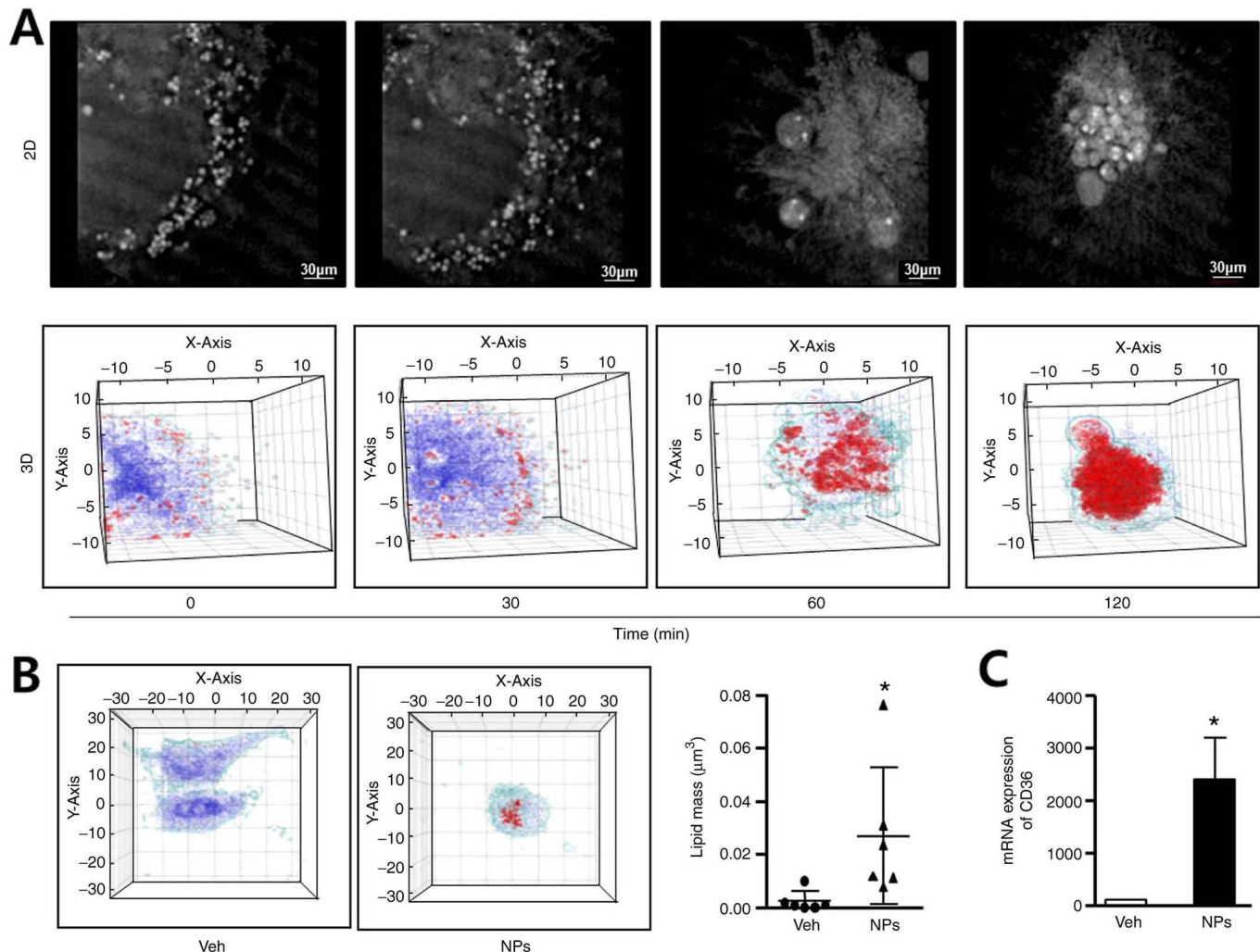

Figure 4. Effect of AS-DK143-NPs on lipid metabolism in HeLa cells. (A) After treating HeLa cells with $30 \mu$ M AS-DK143-NPs, the cellular lipids were observed in real time at $30 \mathrm{~min}, 60 \mathrm{~min}$ and $120 \mathrm{~min}$. Lipid accumulation was evaluated using 3D holographic microscopy. (B) After $24 \mathrm{~h}$ of treatment, lipid mass in HeLa cells was analyzed. Red marks indicate the lipids. Bar graph indicates the lipid mass:cell mass ratio. (C) Relative CD36 mRNA expression evaluated by reverse transcription quantitative PCR. * $\mathrm{P}<0.05$ vs. untreated group. 2D, 2 dimensions; 3D, 3 dimensions; Veh, vehicle; NPs, nanoparticles; AS, aspirin.

with AS-DK143-NPs (Fig. 4B). As presented in Fig. 4C, AS-DK143-NPs significantly increased the mRNA level of CD36 compared with the vehicle.

\section{Discussion}

CVC therapy may include a surgical method, depending on the progression of the lesion (25). Doxorubicin can be used to treat CVC and various types of cancer, such as breast and bladder cancer, Kaposi's sarcoma, lymphoma and acute lymphocytic leukemia. However, anthracycline-based anticancer drugs such as doxorubicin can cause toxicity and permanent damage in cardiac cells (26). Identifying effective anticancer drugs with reduced side effects is therefore crucial in the development of anticancer treatment strategy for CVC, which may improve patient survival and enable surgery. Aspirin was the first synthetic antipyretic and anti-inflammatory analgesic and was used for various purposes, including reducing cardiovascular risk, especially myocardial infarction and ischemic stroke $(27,28)$. DK143 is synthesized from selected plant-derived anticancer drugs (29). In the present study, we attempted to synthesize NPs carrying both compounds, aspirin and DK143, which have excellent anticancer properties and no adverse side effects on the heart (22).

The results from the present study demonstrated that AS-DK143-NPs may have a stronger cytotoxic effect on HeLa cells compare with other human cancer cell lines, including HepG2, BXPC-3 and AGS cell lines. We also determined whether AS-DK143-NPs could induce cell cycle regulation in
HeLa cells. Ki-67 is closely related to tumor cell proliferation. Thus, it is not expressed during the stationary phase (G0) of the cell cycle, but is expressed during the proliferative phases (G1, S, G2 and M phases) (30). The present study demonstrated that AS-DK143-NPs reduced Ki-67 expression. However, these results raised the question of whether AS-DK143-NPs may exert an anticancer effect by inducing apoptosis.

Apoptosis is induced by cellular signals that originate from mitochondria (31). BAX and $\mathrm{Bcl}-2$ play important regulatory roles in apoptosis induction (32). An increased BAX/Bcl-2 expression ratio has been reported to promote apoptotic signals triggered by anticancer drugs (33). In the present study, we observed that apoptosis induced by AS-DK143-NPs could inhibit HeLa cell viability. Furthermore, apoptosis induction by AS-DK143-NPs was confirmed by performing TUNEL assays and staining cell nuclei with the Hoechst dye. The results from these analyses suggested that AS-DK143-NPs may exert anticancer effects by regulating apoptosis.

Apoptosis may also be induced by various cellular molecules, including lipids (34). Lipid accumulation in cancer cells caused by anticancer drugs can activate apoptosis related signaling cascades, resulting in the development of apoptotic bodies (35). These bodies promote tumor cell death via excessive CD36 expression (36). In the present study, we observed that AS-DK143-NPs induced significant lipid accumulation, CD36 expression and lipid droplet formation in HeLa cells (Fig. 4).

In future studies, in order to investigate how lipid accumulation by AS-DK143-NPs induces apoptosis in HeLa cell, we 
need to confirm that lipid droplet accumulation via high glucose or TGFb2 exposure in cancer cells induces apoptosis directly. Our results indicated that AS-DK143-NPs may represent a potential treatment strategy against CVC. Further investigation on the anticancer effects of AS-DK143-NPs in tumor-bearing animal models, the possible adverse effects on normal cells and comparative studies including negative and positive controls is needed to determine whether AS-DK143-NPs may be considered as a potential anticancer drug that could induce cancer cell apoptosis. Future studies will perform flow cytometry (FACS) analyses for cell cycle analysis and additional western blot analysis, including apoptosis-related proteins (BAX, BCL2 and Caspase 3/7), for more detailed apoptosis evaluation. In addition, we are going to conduct cytochrome $c$ oxidase assay for additional mitochondrial fate evaluation.

\section{Acknowledgements}

Not applicable.

\section{Funding}

This study was supported by the Basic Science Research Program through the National Research Foundation of Korea funded by the Ministry of Science and ICT (grant no. 2021R1I1A1A01049147), the Korea Health Technology R\&D Project through the Korea Health Industry Development Institute funded by the Ministry of Health \& Welfare (grant no. HI18C2383), Republic of Korea, the Korea Basic Science Institute (KBSI) under the R\&D programs (grant no. D110710) supervised by the Ministry of Science and ICT. This work was supported by the Technology Development Program (grant no. S3054194) funded by the Korean Ministry of SMEs and Startup.

\section{Availability of data and materials}

The datasets used and/or analyzed during the current study are available from the corresponding author on reasonable request.

\section{Authors' contributions}

KPL, SB, KSP and BSM conceived and designed the study. MSY, KPL, SB and JSP performed the experiments. BSH, SJL, SJO, DHL, RL and SHK analyzed the data. KPL and SB drafted the initial manuscript. KSP and BSM revised the manuscript and confirmed the authenticity of all the raw data. All authors have read and approved the final manuscript.

\section{Ethics approval and consent to participate}

Not applicable.

\section{Patient consent for publication}

Not applicable.

\section{Competing interests}

The authors declare that they have no competing interests.

\section{References}

1. Arbyn M, Weiderpass E, Bruni L, de Sanjosé S, Saraiya M, Ferlay J and Bray F: Estimates of incidence and mortality of cervical cancer in 2018: A worldwide analysis. Lancet Glob Health 8: e191-e203, 2020.

2. Maine D, Hurlburt S and Greeson D: Cervical cancer prevention in the 21st century: Cost is not the only issue. Am J Public Health 101: 1549-1555, 2011.

3. Lowndes CM: Vaccines for cervical cancer. Epidemiol Infect 134: 1-12, 2006.

4. Burd EM: Human papillomavirus and cervical cancer. Clin Microbiol Rev 16: 1-17, 2003.

5. Yim EK and Park JS: The role of HPV E6 and E7 oncoproteins in HPV-associated cervical carcinogenesis. Cancer Res Treat 37: 319-324, 2005.

6. Thomas M, Pim D and Banks L: The role of the E6-p53 interaction in the molecular pathogenesis of HPV. Oncogene 18: 7690-7700, 1999.

7. Fischer M, Uxa S, Stanko C, Magin TM and Engeland K: Human papilloma virus E7 oncoprotein abrogates the p53-p21-DREAM pathway. Sci Rep 7: 2603, 2017.

8. Fan X, Liu Y and Chen JJ: Down-regulation of p21 contributes to apoptosis induced by HPV E6 in human mammary epithelial cells. Apoptosis 10: 63-73, 2005.

9. Photopulos GJ: Surgery or radiation for early cervical cancer. Clin Obstet Gynecol 33: 872-882, 1990.

10. Kumar L and Gupta S: Integrating Chemotherapy in the Management of Cervical Cancer: A Critical Appraisal. Oncology 91 (Suppl 1): 8-17, 2016.

11. Hoffman MS, Roberts WS, Bryson SC, Kavanagh JJ Jr, Cavanagh D and Lyman GH: Treatment of recurrent and metastatic cervical cancer with cis-platin, doxorubicin, and cyclophosphamide. Gynecol Oncol 29: 32-36, 1988.

12. Zhu H, Luo H, Zhang W, Shen Z, Hu X and Zhu X: Molecular mechanisms of cisplatin resistance in cervical cancer. Drug Des Devel Ther 10: 1885-1895, 2016.

13. Serkies K and Jassem J: Concurrent weekly cisplatin and radiotherapy in routine management of cervical cancer: A report on patient compliance and acute toxicity. Int $\mathbf{J}$ Radiat Oncol Biol Phys 60: 814-821, 2004.

14. Sarosiek KA, Ni Chonghaile T and Letai A: Mitochondria: Gatekeepers of response to chemotherapy. Trends Cell Biol 23: 612-619, 2013.

15. Brunelle JK and Letai A: Control of mitochondrial apoptosis by the Bcl-2 family. J Cell Sci 122: 437-441, 2009.

16. Willems PH, Rossignol R, Dieteren CE, Murphy MP and Koopman WJ: Redox Homeostasis and Mitochondrial Dynamics. Cell Metab 22: 207-218, 2015.

17. Jana BA, Chintamaneni PK, Krishnamurthy PT, Wadhwani A and Mohankumar SK: Cytosolic lipid excess-induced mitochondrial dysfunction is the cause or effect of high fat diet-induced skeletal muscle insulin resistance: A molecular insight. Mol Biol Rep 46: 957-963, 2019.

18. Wong A, Chen S, Yang LK, Kanagasundaram Y and Crasta K: Lipid accumulation facilitates mitotic slippage-induced adaptation to anti-mitotic drug treatment. Cell Death Discov 4: 109, 2018.

19. Yang L, Zhu H, Liu D, Liang S, Xu H, Chen J, Wang X and Xu Z: Aspirin suppresses growth of human gastric carcinoma cell by inhibiting survivin expression. J Biomed Res 25: 246-253, 2011.

20. Sostres C, Gargallo CJ and Lanas A: Aspirin, cyclooxygenase inhibition and colorectal cancer. World J Gastrointest Pharmacol Ther 5: 40-49, 2014

21. Johnson KE, Ceglowski JR, Roweth HG, Forward JA, Tippy MD, El-Husayni S, Kulenthirarajan R, Malloy MW, Machlus KR, Chen WY, et al: Aspirin inhibits platelets from reprogramming breast tumor cells and promoting metastasis. Blood Adv 3: 198-211, 2019.

22. Lee DY, Lee KP, Baek S, Park JS, Kim YJ, Kim KN, Kim SR, Yoon MS: Anti-breast cancer activity of aspirin-conjugated chalcone polymeric micelles. Macromol Res 29: 105-110, 2021.

23. Reid Y, Storts D, Riss T, Minor L: Authentication of Human Cell Lines by STR DNA Profiling Analysis. In: Assay Guidance Manual. [Internet]. Markossian S, Grossman A, Brimacombe K, Arkin M, Auld D, Austin CP, Baell J, Chang TDY, Coussens NP, Dahlin JL, et al (eds). Eli Lilly \& Company and the National Center for Advancing Translational Sciences, Bethesda, MD, 2004.

24. Livak KJ and Schmittgen TD: Analysis of relative gene expression data using real-time quantitative PCR and the 2(-Delta Delta C(T)) Method. Methods 25: 402-408, 2001.

25. Sarenac T and Mikov M: Cervical Cancer, Different Treatments and Importance of Bile Acids as Therapeutic Agents in This Disease. Front Pharmacol 10: 484, 2019. 
26. Cai F, Luis MAF, Lin X, Wang M, Cai L, Cen C and Biskup E: Anthracycline-induced cardiotoxicity in the chemotherapy treatment of breast cancer: Preventive strategies and treatment. Mol Clin Oncol 11: 15-23, 2019.

27. Vane JR and Botting RM: The mechanism of action of aspirin. Thromb Res 110: 255-258, 2003.

28. Ittaman SV, VanWormer JJ and Rezkalla SH: The role of aspirin in the prevention of cardiovascular disease. Clin Med Res 12: $147-154,2014$

29. Lee DH, Jung Jung Y, Koh D, Lim Y, Lee YH and Shin SY: A synthetic chalcone, 2'-hydroxy-2,3,5'-trimethoxychalcone triggers unfolded protein response-mediated apoptosis in breast cancer cells. Cancer Lett 372: 1-9, 2016

30. Li LT, Jiang G, Chen Q and Zheng JN: Ki67 is a promising molecular target in the diagnosis of cancer (review). Mol Med Rep 11: 1566-1572, 2015.

31. Indran IR, Tufo G, Pervaiz S and Brenner C: Recent advances in apoptosis, mitochondria and drug resistance in cancer cells. Biochim Biophys Acta 1807: 735-745, 2011

32. Khanzadeh T, Hagh MF, Talebi M, Yousefi B, Azimi A, Hossein Pour Feizi AA and Baradaran B: Investigation of BAX and BCL2 expression and apoptosis in a resveratrol- and prednisolone-treated human T-ALL cell line, CCRF-CEM. Blood Res 53: 53-60, 2018.
33. Naseri MH, Mahdavi M, Davoodi J, Tackallou SH, Goudarzvand $\mathrm{M}$ and Neishabouri $\mathrm{SH}$ : Up regulation of Bax and down regulation of $\mathrm{Bcl} 2$ during 3-NC mediated apoptosis in human cancer cells. Cancer Cell Int 15: 55, 2015.

34. Su LJ, Zhang JH, Gomez H, Murugan R, Hong X, Xu D, Jiang F and Peng ZY: Reactive Oxygen Species-Induced Lipid Peroxidation in Apoptosis, Autophagy, and Ferroptosis. Oxid Med Cell Longev. October 13, 2019. (Epub ahead of print). doi: $10.1155 / 2019 / 5080843$.

35. Cruz ALS, Barreto EA, Fazolini NPB, Viola JPB and Bozza PT: Lipid droplets: Platforms with multiple functions in cancer hallmarks. Cell Death Dis 11: 105, 2020.

36. Frank AC, Ebersberger S, Fink AF, Lampe S, Weigert A, Schmid T, Ebersberger I, Syed SN and Brüne B: Apoptotic tumor cell-derived microRNA-375 uses CD36 to alter the tumor-associated macrophage phenotype. Nat Commun 10: 1135, 2019.

cC) () $९$ This work is licensed under a Creative Commons Attribution-NonCommercial-NoDerivatives 4.0 International (CC BY-NC-ND 4.0) License. 